\title{
MÖBIUS TRANSFORMATIONS OF THE DISC AND ONE-PARAMETER GROUPS OF ISOMETRIES OF $H^{p}$
}

BY

\author{
EARL BERKSON, ROBERT KAUFMAN AND HORACIO PORTA( $\left.{ }^{1}\right)$
}

ABSTRACT. Let $\left\{T_{t}\right\}$ be a strongly continuous one-parameter group of isometries in $H^{p} \quad(1 \leqslant p<\infty, p \neq 2)$ with unbounded generator. There is a uniquely determined one-parameter group $\left\{\phi_{t}\right\}, t \in R$, of Möbius transformations of the (open) disc $D$ corresponding to $\left\{T_{t}\right\}$ by way of Forelli's theorem. The interplay between $\left\{T_{t}\right\}$ and $\left\{\phi_{t}\right\}$ is studied, and the spectral properties of the generator $A$ of $\left\{T_{t}\right\}$ are analyzed in this context. The nature of the set $S$ of common fixed points of the functions $\phi_{t}$ plays a crucial role in determining the behavior of $A$. The spectrum of $A$, which is a subset of $i R$, can

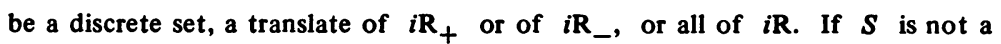
doubleton subset of the unit circle, $\left\{T_{t}\right\}$ can be extended to a holomorphic semigroup of $H^{p}$-operators, the semigroup being defined on a half-plane. The treatment of $\left\{r_{t}\right\}$ is facilitated by developing appropriate properties of oneparameter groups of Möbius transformations of $D$. In particular, such groups are in one-to-one correspondence (via an initial-value problem) with the nonzero polynomials $q$, of degree at most 2 , such that $\operatorname{Re}[\bar{z} q(z)]=0$ for all unimodular z. $A$ has an explicit description (in terms of the polynomial corresponding to $\left\{\phi_{t}\right\}$ ) as a differential operator.

0. Introduction. The purpose of this paper is to investigate strongly continuous one-parameter groups of isometries (and their generators) in the spaces $H^{p}$ (of the disc), $1 \leqslant p<\infty$. Such groups were studied in [1], where basic facts were developed. The following is of central importance to our subject [4, Theorem 2].

(0.1) Proposition. If $T$ is a linear isometry of $H^{p}$ onto $H^{p}, 1 \leqslant$ $p<\infty, p \neq 2$, then there are a unimodular constant $\nu$ and a Möbius transformation of the disc $\phi$ such that

$$
(T f)(z)=\nu\left[\phi^{\prime}(z)\right]^{1 / p} f(\phi(z)), \quad f \in H^{p},|z|<1 .
$$

Received by the editors December 18, 1973.

AMS (MOS) subject classifications (1970). Primary 46E15, 47A10, 47D05.

Key words and phrases. Isometry, $H^{p}$, Möbius transformation, group, generator, spectrum.

(1) The work of the first and third authors was supported by a National Science Foundation grant. The second author was supported by a Sloan Fellowship. 
On the other hand, if $1 \leqslant p<\infty,(0.2)$ defines a linear isometry of $H^{p}$ onto $H^{p}$.

In $\S 2$ we take up the relationship between groups of $H^{p}$-isometries and one-parameter groups of Möbius transformations of $D(=\{z \in \mathrm{C}:|z|<1\})$. A one-parameter group of Möbius transformations of the disc is a homomorphism $t \mapsto \phi_{t}$ of the additive group of $\mathbf{R}$ into the group $M$ (under composition) of all univalent analytic mappings of $D$ onto $D$ such that, for each $z_{0} \in D$, $\phi_{t}\left(z_{0}\right)$ is a continuous function of $t$ and, for some $u \in \mathbf{R}, \phi_{u}$ is not the identity map.

One-parameter groups of Möbius transformations of $D$ are closely associated with polynomials of a certain type, and this association is developed in $\S 1$ (see Theorem (1.5)). $\S 1$ also deals with the classification of such groups, as well as their extensions to groups of linear fractional transformations (see Theorem (1.10)).

In $\S \S 3$ and 4 we consider the generators of one-parameter groups of $H^{p}$. isometries, and present a fairly comprehensive treatment of the spectral properties of such a generator.

Throughout what follows, we shall denote the unit circle $\{z \in \mathrm{C}:|z|=1\}$ by $C$, the extended complex plane by $\mathbf{C}_{e}$, and the composition of two mappings $f$ and $g$ by $f \circ g$. We usually deal with $H^{p}(D)$. When, on occasion, we pass. to the boundary $C$, this fact will be made clear by the context.

We remark that for $1 \leqslant p<\infty, p \neq 2$, it is known [1, Theorem (2.8)] that the one-parameter groups of $H^{p}$-isometries continuous in the uniform operator topology are precisely the trivial groups of the form $\left\{e^{i \beta t} I\right\}, t \in \mathbf{R}$, where $I$ is the identity operator, and $\beta$ is a real constant.

1. One-parameter groups of Möbius transformations of the disc and their extensions. Let $\left\{\phi_{t}\right\}, t \in \mathbf{R}$, be a one-parameter group of Möbius transformations of $D$. It will sometimes be convenient to write $\phi(t, z)$ in place of $\phi_{t}(z)$ (for $t \in \mathbf{R}, z \in \mathbf{C}$ ). In this notation, the partial derivative at $\left(r, z_{0}\right)$ of $\phi(\cdot, \cdot)$ with respect to $t$ (resp., with respect to $z$ ) will be denoted by $\phi_{1}\left(r, z_{0}\right)$ (resp., $\left.\phi_{2}\left(r, z_{0}\right)\right)$, provided it exists. As observed in [1, §1], $\phi_{1}\left(r, z_{0}\right)$ and $\phi_{2}\left(r, z_{0}\right)$ exist provided $z_{0}$ is not a pole of the Möbius transformation $\phi_{r}(\cdot)$. The following fact will be basic to our considerations [1, Proposition (1.5)] :

(1.1) Scholium. The set $S$ of common fixed points in $\mathbf{C}_{e}$ of the functions $\phi_{t}, t \in \mathbf{R}$, must be one of the following: (i) a doubleton set consisting of a point $\tau$ in $D$ and of $\bar{\tau}^{-1}$ (the latter taken to be $\infty$ if $\tau=0$ ), (ii) $a$ singleton subset of $C$, or (iii) a doubleton subset of $C$. If $u \in \mathbf{R}$ and $\phi_{u}(\cdot)$ is not the identity map, then $S$ coincides with the set of all fixed points in $\mathbf{C}_{e}$ of $\phi_{u}(\cdot)$. 
As in [1], $\left\{\phi_{t}\right\}$ will be said to be of type (i), (ii), or (iii) according as (i), (ii), or (iii) of the mutually exclusive descriptions for $S$ in (1.1) holds. A further basic fact from $[1,(1.6)$ and $(1.7)]$ is that $\phi_{1}(0, z)$ is a nonzero polynomial in $z$ of degree one or two whose set of zeros is $S \cap C$. We denote $\phi_{1}(0, \cdot)$ by $q(\cdot)$ and call it the invariance polynomial of $\left\{\phi_{t}\right\}, t \in \mathbf{R}$.

We shall now characterize one-parameter groups of Möbius transformations of $D$ by a suitable type of initial-value problem. For such a group $\left\{\phi_{t}\right\}$, $|\phi(t, z)|^{2}=1$ for $t \in \mathbf{R}, z \in C$. If we partially differentiate this identity with respect to $t$ and then set $t=0$, we get

$$
\operatorname{Re}[\bar{z} q(z)]=0 \text { for all } z \text { in } C \text {. }
$$

Let $I$ be the set of all nonzero polynomials $f$ of degree at most 2 such that $\operatorname{Re}[\bar{z} f(z)]=0$ for all unimodular $z$. A simple calculation based on the definition and the linear independence on $C$ of the functions $z, 1$, and $\bar{z}$ shows that $I$ consists of all polynomials $f$ of the form $f(z)=A z^{2}+i B z-\bar{A}$, where $A$ is a complex constant, $B$ is a real constant, and $|A|^{2}+B^{2}>0$.

Next we observe that for the group $\left\{\phi_{t}\right\}$ we have for all $s, t \in \mathbf{R}$ and $z \in \bar{D}, \phi(s+t, z)=\phi(s, \phi(t, z))$. Partial differentiation with respect to $s$, followed by setting $s=0$, gives $\phi_{1}(t, z)=q(\phi(t, z))$ for $t \in \mathbf{R}, z \in \bar{D}$.

Conversely, let $p_{0} \in I$, let $J$ be an interval of $\mathbf{R}$ containing 0 , and consider the initial-value problem on $J \times \bar{D}$

$$
\partial \psi(t, z) / \partial t=p_{0}(\psi(t, z)), \quad \psi(0, z)=z .
$$

Regarding (1.3) as an ordinary differential equation for each fixed $z \in \bar{D}$, we get from a standard uniqueness theorem that (1.3) has at most one solution for $\psi$ on $J \times \bar{D}$, and the Picard method of successive approximations shows that there is a $\delta>0$ such that (1.3) has a solution on $[-\delta, \delta] \times \bar{D}$ which is the uniform limit on $[-\delta, \delta] \times \bar{D}$ of a sequence of polynomials in $t$ and $z$. Moreover, if $\alpha>0$, and $\psi$ is a solution of (1.3) on $[-\alpha, \alpha] \times \bar{D}$, then for fixed $z_{0} \in \bar{D}$

$$
\frac{d}{d t}\left[\left|\psi\left(t, z_{0}\right)\right|^{2}-1\right]=2 \operatorname{Re}\left[\overline{\psi\left(t, z_{0}\right)} p_{0}\left(\psi\left(t, z_{0}\right)\right)\right] \text { on }[-\alpha, \alpha]
$$

For $w \in \mathrm{C}$ let $w^{*}$ be $w /|w|$ if $w \neq 0$, and 1 if $w=0$. Then

$$
p_{0}\left(\psi\left(t, z_{0}\right)\right)-p_{0}\left(\psi\left(t, z_{0}\right)^{*}\right)=O\left(\left|\psi\left(t, z_{0}\right)-\psi\left(t, z_{0}\right)^{*}\right|\right) \text { on }[-\alpha, \alpha] \text {. }
$$

Thus (1.4) and the definition of $I$ give us that

$$
\left|\frac{d}{d t}\left[\left|\psi\left(t, z_{0}\right)\right|^{2}-1\right]\right|=O\left(\left.|| \psi\left(t, z_{0}\right)\right|^{2}-1 \mid\right) .
$$

A standard Gronwall type argument allows us to conclude from this fact that, for $t \in[-\alpha, \alpha],|\psi(t, z)| \leqslant 1$ (resp., $|\psi(t, z)|=1$ ) for $z \in \bar{D}$ (resp., for $|z|=1$ ). 
It is easy to see with the aid of this boundedness conclusion that (1.3) has a unique solution $\phi$ on $\mathbf{R} \times \bar{D}$. As usual, for $t \in \mathbf{R}$ we write $\phi_{t}$ for the function $\phi(t, \cdot)$ on $\bar{D}$. In particular each $\phi_{t}$ maps $\bar{D}$ into $\bar{D}$ and $C$ into $C$. Since the equation in (1.3) is autonomous, we get that $\phi_{t+s}(z)=\phi_{t}\left(\phi_{s}(z)\right)$ for $t, s \in \mathbf{R}, z \in \bar{D}$. Our earlier observation concerning the Picard method assures us of an interval $[-\delta, \delta]$ such that, for each $t$ in $[-\delta, \delta], \phi_{t}$ is continuous on $\bar{D}$ and analytic on $D$. It is now clear that, for $t \in[-\delta, \delta], \phi_{t}$ is a oneto-one analytic map of $D$ onto $D$ and must be a Möbius transformation of the disc. Since $\phi_{u+v}=\phi_{u} \circ \phi_{v}$ for all $u, v \in \mathbf{R}$, it follows easily that $\phi_{u}$ is a Möbius transformation of the disc for each $u \in \mathbf{R}$. Moreover, it is immediate from (1.3) that $p_{0}$ is the invariance polynomial of the one-parameter group $\left\{\phi_{t}\right\}$ of Möbius transformations of the disc. We have established:

(1.5) THEOREM. The correspondence which assigns to each one-parameter group of Möbius transformations of the disc its invariance polynomial is a oneto-one map of the set of all such groups onto 1 . The inverse of this map assigns to each $p_{0} \in I$ the unique solution on $\mathbf{R} \times \bar{D}$ of the initial-value problem

$$
\partial \psi(t, z) / \partial t=p_{0}(\psi(t, z)), \quad \psi(0, z)=z .
$$

The next theorem makes explicit the correspondence of Theorem (1.5). Some additional notation will be helpful. Let $\mathbf{R}_{\mathbf{0}}$ (resp., $P$ ) denote the set of nonzero (resp., positive) real numbers. Let $S_{1}$ (resp., $S_{2}$ ) be the Cartesian product $\mathbf{R}_{0} \times D$ (resp., $\mathbf{R}_{0} \times C$ ). Let $S_{3}$ be the set of all ordered triples $(c, \alpha, \beta)$ such that $c \in P, \alpha \in C, \beta \in C, \alpha \neq \beta$. Let $G_{1}$ (resp., $G_{2}, G_{3}$ ) be the set of all one-parameter groups of Möbius transformations of the disc of type (i) (resp., type (ii), type (iii)). For $\tau \in D$, let $\gamma_{\tau}$ be the Möbius transformation of the disc given by $\gamma_{\tau}(z)=(z-\tau) /(\overline{\tau z}-1)$. (Note that $\gamma_{\tau}$ is its own inverse map.) For $(\alpha, \beta) \in C \times C$ with $\alpha \neq \beta$, let $\sigma_{\alpha, \beta}$ be the linear fractional transformation given by $\sigma_{\alpha, \beta}(z)=(z-\alpha) /(z-\beta)$.

(1.6) THEOREM. (i) To each ordered pair $(c, \tau)$ in $S_{1}$ there corresponds a group $\left\{\phi_{t}\right\}$ belonging to $G_{1}$ given by

$$
\phi_{t}(z)=\gamma_{\tau}\left(e^{i c t} \gamma_{\tau}(z)\right) \text { for } t \in \mathbf{R}, z \in D .
$$

This correspondence is a one-to-one map of $S_{1}$ onto $G_{1}$. If $\left\{\phi_{t}\right\} \in G_{1}$ has the unique representation (1.7), then its invariance polynomial is icz (resp., ic $\left.\bar{\tau}\left(|\tau|^{2}-1\right)^{-1}(z-\tau)(z-1 / \bar{\tau})\right)$ if $\tau=0$ (resp., $\left.\tau \neq 0\right)$.

(ii) To each ordered pair $(c, \alpha)$ in $S_{2}$ there corresponds a group $\left\{\phi_{t}\right\}$ belonging to $G_{2}$ given by 


$$
\phi_{t}(z)=\frac{(1-i c t) z+i c t \alpha}{-i c \bar{\alpha} t z+(1+i c t)} \quad \text { for } t \in \mathbf{R}, z \in D \text {. }
$$

This correspondence is a one-to-one map of $S_{2}$ onto $G_{2}$. If $\left\{\phi_{t}\right\} \in G_{2}$ has the unique representation (1.8), then its invariance polynomial is ica $(z-\alpha)^{2}$.

(iii) To each ordered triple $(c, \alpha, \beta)$ in $S_{3}$ there corresponds a group $\left\{\phi_{t}\right\}$ belonging to $G_{3}$ given by

$$
\phi_{t}(z)=\left(\sigma_{\alpha, \beta}\right)^{-1}\left(e^{c t} \sigma_{\alpha, \beta}(z)\right) \quad \text { for } t \in \mathbf{R}, z \in D .
$$

This correspondence is a one-to-one map of $S_{3}$ onto $G_{3}$. If $\left\{\phi_{t}\right\} \in G_{3}$ has the unique representation (1.9), then its invariance polynomial is $(c /(\alpha-\beta))(z-\alpha)(z-\beta)$.

Proof. We begin with (iii). Let $(c, \alpha, \beta) \in S_{3}$. Note that $\sigma_{\alpha, \beta}(\bar{D})$ is the half-plane $H$ (including the point at $\infty$ ) given by $\operatorname{Re}[(1-\bar{\alpha} \beta) z] \leqslant 0$. Thus if we define $\phi_{t}$ by (1.9), then $\phi_{t}$ is a linear fractional transformation with $\phi_{t}(\bar{D})=\left(\sigma_{\alpha, \beta}\right)^{-1}(H)=\bar{D}$. Clearly $\left\{\phi_{t}\right\}, t \in \mathbf{R}$, is a one-parameter group of Möbius transformations of the disc having $\alpha$ and $\beta$ as common fixed points. Conversely, let $\left\{\phi_{t}\right\}$ be of type (iii) with distinct common fixed points $\alpha, \beta \in$ $C$. It follows from elementary facts about linear fractional transformations [7, p. 323] that for each $t \in \mathbf{R}$ there is a unique nonzero complex constant $K_{t}$ such that $\sigma_{\alpha, \beta}\left(\phi_{t}(z)\right)=K_{t} \sigma_{\alpha, \beta}(z)$. Moreover, $K_{t+s}=K_{t} K_{s}$ for all $t, s \in \mathbf{R}$. Since $\phi_{t}(z)$ is continuous in $t$ for each $z \in D, K_{t}$ is a continuous function of $t$. If $H$ is the half-plane mentioned above, then for each $t \in \mathbf{R}, H=K_{t} H$, which implies $K_{t}>0$. It is now clear that there is a real number $\lambda$ such that $K_{t}=e^{\lambda t}$ for all $t \in \mathbf{R}$. Since $\left\{\phi_{t}\right\}$ is not a constant function of $t, \lambda \neq 0$. By taking reciprocals, if necessary, on both sides of the equation

$$
\frac{\phi_{t}(z)-\alpha}{\phi_{t}(z)-\beta}=e^{\lambda t} \frac{z-\alpha}{z-\beta}, \quad t \in \mathbf{R}, \quad z \in D,
$$

we conclude that $\left\{\phi_{t}\right\}$ corresponds to an ordered triple in $S_{3}$. If $\left(c_{1}, \alpha_{1}, \beta_{1}\right)$ and $\left(c_{2}, \alpha_{2}, \beta_{2}\right)$ belong to $S_{3}$ and define the same group $\left\{\phi_{t}\right\}$ by (1.9), then it is obvious from (1.9) that the set of common fixed points of $\left\{\phi_{t}\right\}, t \in \mathbf{R}$, coincides with each of the sets $\left\{\alpha_{1}, \beta_{1}\right\}$ and $\left\{\alpha_{2}, \beta_{2}\right\}$. If $\alpha_{1}=\beta_{2}$ and $\beta_{1}=\alpha_{2}$, then it follows readily that $e^{-c_{2} t}=e^{c_{1} t}$ for all $t \in \mathbf{R}$, which gives the conclusion $-c_{2}=c_{1}$. But $c_{1}>0$ and $c_{2}>0$. Thus $\alpha_{1}=\alpha_{2}$ and $\beta_{1}=$ $\beta_{2}$, whence $c_{1}=c_{2}$. To compute the invariance polynomial of $\left\{\phi_{t}\right\}$ in (1.9), we first differentiate with respect to $t$ on both sides of the equation $\sigma_{\alpha, \beta}\left(\phi_{t}(z)\right)=e^{c t} \sigma_{\alpha, \beta}(z)$ to get

$$
\sigma_{\alpha, \beta}^{\prime}(\phi(t, z)) \partial \phi(t, z) / \partial t=c e^{c t} \sigma_{\alpha, \beta}(z) .
$$

Dividing the latter equation by the former gives 


$$
\frac{\sigma_{\alpha, \beta}^{\prime}(\phi(t, z))}{\sigma_{\alpha, \beta}(\phi(t, z))} \frac{\partial \phi(t, z)}{\partial t}=c
$$

By direct calculation,

$$
\frac{\sigma_{\alpha, \beta}(z)}{\sigma_{\alpha, \beta}^{\prime}(z)}=\frac{1}{\alpha-\beta}(z-\alpha)(z-\beta) \text { for } z \in D
$$

Thus

$$
\frac{\partial \phi(t, z)}{\partial t}=\frac{c}{\alpha-\beta}(\phi(t, z)-\alpha)(\phi(t, z)-\beta) \quad \text { for } z \in D
$$

and the desired conclusion follows. The proof of (i) (except for the trivial parts when $\tau=0$ ) is entirely analogous to that of (iii) (using $\tau$ and $\bar{\tau}^{-1}$ in place of $\alpha$ and $\beta$ ). Also, (i) has been tabulated in [1, Theorem (1.10) and Corollary (1.13)] , and we omit the details of its proof. It remains to establish (ii). Obviously the invariance polynomials of the groups of type (ii) are precisely the polynomials belonging to $I$ with a double root belonging to $C$. The latter are, in turn, precisely the polynomials of the form $q(z)=i c \bar{\alpha}(z-\alpha)^{2}$ for $c$ real and nonzero and $\alpha \in C$. The proof of (ii) is now easily completed by explicitly solving, for such a polynomial $q$, the initial-value problem of Theorem (1.5), thereby obtaining the corresponding group, $\left\{\phi_{t}\right\}, t \in \mathbf{R}$. The solution guaranteed by Theorem (1.5) can be found on $R \times D$ by separation of variables.

In the next section we shall be concerned with extending, whenever possible, a one-parameter group of isometries of $H^{p}$ to a semigroup (defined on a halfplane) of operators on $H^{p}$. In preparation for this we now take up the related problem of extending a one-parameter group of Möbius transformations of the disc to a planar group of linear fractional transformations in the following sense.

Definition. A planar group $\left\{L_{w}\right\}, w \in \mathbf{C}$, of linear fractional transformations is a homomorphism $w \mapsto L_{w}$ of the additive group of $\mathbf{C}$ into the group (under composition) of all linear fractional transformations such that, for each $z \in \mathbf{C}, w \rightarrow L_{w}(z)$ is a continuous mapping of $\mathbf{C}$ into $\mathbf{C}_{e}$, for some $w$ $L_{w}$ is not the identity map, and, for each $z \in \mathbf{C}, d L_{w}(z) /\left.d w\right|_{w=0}$ exists. The planar group $\left\{L_{w}\right\}, w \in \mathbf{C}$, is said to extend a one-parameter group $\left\{\phi_{t}\right\}$, $t \in \mathbf{R}$, of Möbius transformations of the disc provided $L_{t}=\phi_{t}$ for $t \in \mathbf{R}$.

(1.10) THEOREM. Every one-parameter group $\left\{\phi_{t}\right\}$ of Möbius transformations of the disc can be uniquely extended to a planar group $\left\{\Phi_{w}\right\}, w \in \mathbf{C}$, of linear fractional transformations. In fact:

(i) If $\left\{\phi_{t}\right\}$ is of type (i) with the representation (1.7), then $\left\{\Phi_{w}\right\}, w \in$ C, has the form 


$$
\Phi_{w}(z)=\gamma_{\tau}\left(e^{i c w} \gamma_{\tau}(z)\right)=\frac{\left(e^{i c w}-|\tau|^{2}\right) z+\tau\left(1-e^{i c w}\right)}{\tau\left(e^{i c w}-1\right) z+1-|\tau|^{2} e^{i c w}}
$$

(ii) If $\left\{\phi_{t}\right\}$ is of type (ii) with the representation (1.8), then $\left\{\Phi_{w}\right\}$, $w \in \mathbf{C}$, has the form

$$
\Phi_{w}(z)=\frac{(1-i c w) z+i c w \alpha}{-i c \bar{w} w z+1+i c w}
$$

(iii) If $\left\{\phi_{t}\right\}$ is of type (iii) with the representation (1.9), then $\left\{\Phi_{w}\right\}$, $\boldsymbol{w} \in \mathbf{C}$, has the form

$$
\Phi_{w}(z)=\left(\sigma_{\alpha, \beta}\right)^{-1}\left(e^{c w} \sigma_{\alpha, \beta}(z)\right)=\frac{\left(\beta e^{c w}-\alpha\right) z+\alpha \beta\left(1-e^{c w}\right)}{\left(e^{c w}-1\right) z+\beta-\alpha e^{c w}}
$$

Proof. One verifies readily that the middle and right-hand members in each of (1.11) and (1.13) are equal, and that (1.12) defines a planar group $\left\{\Phi_{w}\right\}$, $w \in \mathbf{C}$, which obviously extends the group $\left\{\phi_{t}\right\}$ in (ii). It is obvious that (1.11) (resp., (1.13)) defines a planar group extending the group $\left\{\phi_{t}\right\}$ in (i) (resp., (iii)). To prove uniqueness, let $\left\{\phi_{t}\right\}$ be a one-parameter group of Möbius transformations of $D$ with invariance polynomial $q$, and let $\left\{\Psi_{w}\right\}$ be a planar group of linear fractional transformations extending $\left\{\phi_{t}\right\}$. It follows from definitions that if $\left(w_{0}, z_{0}\right) \in \mathbf{C} \times \mathbf{C}$ and $z_{0}$ is not a pole of $\Psi_{w_{0}}$, then $d \Psi_{w}\left(z_{0}\right) /\left.d w\right|_{w=w_{0}}$ exists and equals $q\left(\Psi_{w_{0}}\left(z_{0}\right)\right)$. Thus if $v_{0} \in \mathbf{R}$ and $z_{0}$ is not a pole of $\Psi_{i v_{0}}$, then $d \Psi_{i v}\left(z_{0}\right) /\left.d v\right|_{v=v_{0}}=i q\left(\Psi_{i v_{0}}\left(z_{0}\right)\right)$. The Picard method and a standard uniqueness theorem show that there is a $\delta>0$ such that for each $z \in \bar{D}$, the initial value problem $d y / d v=i q(y), y(0)=z$, has a unique solution on every subinterval containing 0 of $[-\delta, \delta](\delta$ is independent of $z \in \bar{D}$ ). The desired uniqueness of extension now follows readily.

REMARKS. (1) The uniqueness of the representation for $\left\{\Phi_{w}\right\}$ in (1.11) (resp., (1.12), (1.13)) follows from the uniqueness of the representation for $\left\{\phi_{t}\right\}$ in (1.7) (resp., (1.8), (1.9)).

(2) If $\left\{L_{w}\right\}$ is a planar group of linear fractional transformations, then an argument similar to the one used in proving [1, Proposition (1.5)] shows that for any complex number $w_{0}$ such that $L_{w_{0}}$ is not the identity, the set of fixed points in $\mathbf{C}_{e}$ of $L_{w_{0}}$ coincides with the set of common fixed points in $\mathbf{C}_{e}$ of the functions $L_{w}, w \in \mathbf{C}$. In particular, if $\left\{L_{w}\right\}$ extends a one-parameter group $\left\{\phi_{t}\right\}$ of Möbius transformations of $D$, then, by the last sentence in (1.1), the set of common fixed points in $\mathbf{C}_{e}$ of the functions $L_{w}$ for $w \in \mathbf{C}$ coincides with the set of common fixed points in $\mathbf{C}_{e}$ of the functions $\phi_{t}$ for $t \in \mathbf{R}$. 
(1.14) THEOREM. The linear fractional transformation $\Phi_{w}$ in (1.11) (resp., (1.12), (1.13)) maps $D$ into $D$ if and only if $c \operatorname{Im} w \geqslant 0$ (resp., $c \operatorname{Im} w \leqslant 0, c \operatorname{Im} w=2 k \pi$ for some integer $k$ ).

Proof. By the maximum principle $\Phi_{w}(D) \subseteq D$ if and only if $\Phi_{w}(\bar{D}) \subseteq$ $\bar{D}$. Consider $\Phi_{w}$ in (1.11). Since $\gamma_{\tau}$ is a Möbius transformation of the disc, $\Phi_{w}(D) \subseteq D$ if and only if $e^{i c w} D \subseteq D$. The assertion for this case is established. Consider next the group $\left\{\Phi_{w}\right\}, w \in \mathbf{C}$, in (1.12). For convenience, put the subscript zero on the constant $c$ in (1.12) so that the real constant is now denoted by the symbol $c_{0}$. For arbitrary $\tau$ in $D, w \in \mathrm{C}$, let $\Phi_{w}^{(\tau)}$ be the linear fractional transformation in (1.11) with $c=c_{0}\left(|\tau|^{2}-1\right)$. Let $\left(\delta_{m n}(w)\right)$ be the $2 \times 2$ matrix corresponding to the representation for $\Phi_{w}$ in (1.12), and let $\left(\delta_{m n}^{(\tau)}(w)\right)$ be the matrix corresponding to the representation for $\Phi_{w}^{(\tau)}$ on the far right of (1.11). It is easy to see from the power series expansion for the exponential function that, uniformly on bounded subsets of the $w$-plane, we have, as $\tau \rightarrow \alpha$,

$$
\delta_{m n}^{(\tau)}(w)=\left(1-|\tau|^{2}\right) \delta_{m n}(w)+o\left(1-|\tau|^{2}\right) \text { for } m, n=1,2 .
$$

If $c_{0} \operatorname{Im} w \leqslant 0$ and $z \in \bar{D}$, then the already established first assertion of the theorem insures that $\left|\Phi_{w}^{(\tau)}(z)\right| \leqslant 1$. This fact together with (1.15) enables us to conclude that $\Phi_{w}(\bar{D}) \subseteq \bar{D}$ for $c_{0} \operatorname{Im} w \leqslant 0$. On the other hand, if $w$ is fixed with $c_{0} \operatorname{Im} w>0$, then from (1.11),

$$
\gamma_{\tau}\left(\Phi_{w}^{(\tau)}(C)\right)=\left[\exp \left(c_{0}\left(1-|\tau|^{2}\right) \operatorname{Im} w\right)\right] C .
$$

If $z_{0} \in C$, and $z_{0}$ is not a pole of $\Phi_{w}$, then it follows from (1.15) that for $|\tau-\alpha|$ sufficiently small and positive, $z_{0}$ is not a pole of $\Phi_{w}^{(\tau)}$. Hence

$$
\left|\Phi_{w}^{(\tau)}\left(z_{0}\right)-\tau\right|=\left|\bar{\tau} \Phi_{w}^{(\tau)}\left(z_{0}\right)-1\right| \exp \left(c_{0}\left(1-|\tau|^{2}\right) \operatorname{Im} w\right),
$$

and so, as $\tau \rightarrow \alpha$,

$$
\begin{aligned}
\mid \Phi_{w}^{(\tau)}\left(z_{0}\right)- & \left.\tau\right|^{2}-\left|\bar{\tau} \Phi_{w}^{(\tau)}\left(z_{0}\right)-1\right|^{2} \\
& =2\left|\bar{\tau} \Phi_{w}^{(\tau)}\left(z_{0}\right)-1\right|^{2} c_{0}\left(1-|\tau|^{2}\right) \operatorname{Im} w+o\left(1-|\tau|^{2}\right) .
\end{aligned}
$$

If we simplify (1.16), divide by $\left(1-|\tau|^{2}\right)$, and let $\tau \rightarrow \alpha$, we get

$$
\left|\Phi_{w}\left(z_{0}\right)\right|^{2}-1=2\left|\bar{\alpha} \Phi_{w}\left(z_{0}\right)-1\right|^{2} c_{0} \operatorname{Im} w .
$$

Since $\alpha$ is a fixed point of $\Phi_{w}$, if $z_{0} \neq \alpha$ then the right-hand side of (1.17) is positive. Thus $\Phi_{w}$ does not map $\bar{D}$ into $\bar{D}$ for $c_{0} \operatorname{Im} w>0$.

Finally, for arbitrary $w \in \mathbf{C}$, and the linear fractional transformation $\Phi_{w}$ in (1.13), $\Phi_{w}(\bar{D}) \subseteq \bar{D}$ if and only if $e^{c w} H \subseteq H$, where $H$ is the half-plane 
$\operatorname{Re}[(1-\bar{\alpha} \beta) z] \leqslant 0$. This completes the proof of the theorem.

Let $\left\{\phi_{t}\right\}$ be an arbitrary one-parameter group of Möbius transformations of $D$. In order to expedite the use of $(0.1)$ in the study of one-parameter groups of isometries of $H^{p}$, we note here that $\phi_{2}(\cdot, \cdot)$ has a continuous logarithm $L_{\phi}(\cdot, \cdot)$ on $\mathbf{R} \times \bar{D}$ such that $L_{\phi}(0,0)=0$ (the uniqueness of a logarithm meeting these requirements is immediate from connectedness). Indeed, if we denote $\phi_{2}(t, \cdot)$ (resp., $\phi_{22}(t, \cdot)$ ) by $\phi_{t}^{\prime}(\cdot)$ (resp., $\left.\phi_{t}^{\prime \prime}(\cdot)\right)$, then straightforward reasoning proves the existence of $L_{\phi}(\cdot, \cdot)$ in the form

$$
L_{\phi}(t, z)=\int_{0}^{t} \frac{d\left[\phi_{u}^{\prime}(0)\right]}{d u} \frac{1}{\phi_{u}^{\prime}(0)} d u+\int_{0}^{z} \frac{\phi_{t}^{\prime \prime}(\xi)}{\phi_{t}^{\prime}(\xi)} d \xi \quad \text { for } t \in \mathbf{R}, z \in \bar{D} .
$$

From the connectedness of $\mathbf{R} \times \mathbf{R} \times \bar{D}$ we see that

$$
L_{\phi}(t+s, z)=L_{\phi}\left(t, \phi_{s}(z)\right)+L_{\phi}(s, z) \quad \text { for all } t \in \mathbf{R}, s \in \mathbf{R}, z \in \bar{D} .
$$

If, further, $\left\{\phi_{t}\right\}$ is of type (i) or (ii), then, in the notation of Theorem (1.14), let $H_{\Phi}$ be the half-plane $\left\{w \in \mathbf{C}: \Phi_{w}(\bar{D}) \subseteq \bar{D}\right\}$. In a fashion analogous to the foregoing we note that $\Phi_{2}(w, z)\left(=\partial \Phi_{w}(z) / \partial z\right)$ has, on $H_{\Phi} \times \bar{D}$, a unique continuous logarithm vanishing at $(0,0)$. Denoting this logarithm by $L_{\Phi}$, we have that

$$
\begin{aligned}
& L_{\Phi}(w, z)=\int_{0}^{w} \frac{d\left[\Phi_{\xi}^{\prime}(0)\right]}{d \xi} \frac{1}{\Phi_{\xi}^{\prime}(0)} d \xi+\int_{0}^{z} \frac{\Phi_{w}^{\prime \prime}(\xi)}{\Phi_{w}^{\prime}(\xi)} d \xi \\
& \text { for } w \in H_{\Phi}, z \in \bar{D} .
\end{aligned}
$$

The contour of the first integral on the right of $(1.20)$ is in $H_{\Phi}$. Moreover,

$$
L_{\Phi}\left(w_{1}+w_{2}, z\right)=L_{\Phi}\left(w_{1}, \Phi_{w_{2}}(z)\right)+L_{\Phi}\left(w_{2}, z\right)
$$

$$
\text { for all } w_{1} \in H_{\Phi}, w_{2} \in H_{\Phi}, z \in \bar{D} \text {. }
$$

2. One-parameter groups of isometries of $H^{p}$. Let $G$ be the set of all oneparameter groups of Möbius transformations of $D$. If $\left\{\phi_{t}\right\} \in G$ and $1 \leqslant p<$ $\infty$, then for $t \in \mathbf{R}, z \in \bar{D}$ we define $\left[\phi_{t}^{\prime}(z)\right]^{1 / p}$ to be $\exp \left[(1 / p) L_{\phi}(t, z)\right]$. Also, let $\Gamma_{p}$ be the set of all one-parameter groups of isometries of $H^{p}$ which are continuous in the strong, but not in the uniform, operator topology. For each nonnegative integer $n$, let $e_{n}$ be the function in $H^{\infty}$ defined by $e_{n}(z)=z^{n}$.

(2.1) THEOREM. If $1 \leqslant p<\infty$, there is a one-to-one map $M_{p}$ of $\mathbf{R} \times G$ into $\Gamma_{p}$. The $M_{p}$-image $\left\{T_{t}\right\}$ of $\left(\omega,\left\{\phi_{t}\right\}\right)$ is given by

$$
\left(T_{t} f\right)(z)=e^{i \omega t}\left[\phi_{t}^{\prime}(z)\right]^{1 / p} f\left(\phi_{t}(z)\right) \quad \text { for } f \in H^{p}, z \in D, t \in \mathbf{R} .
$$


If, further, $p \neq 2, M_{p}$ maps $\mathbf{R} \times G$ onto $\Gamma_{p}$.

Proof. It is immediate from (0.1) and (1.19) that $\left\{T_{t}\right\}$ in (2.2) is a oneparameter group of isometries of $H^{p}$. Strong continuity of $\left\{T_{t}\right\}$ is easily verified by first noticing that if $f$ is a polynomial, $T_{t} f$, as a function of $t$, is continuous from $\mathbf{R}$ to $H^{p}$. $\left\{T_{t}\right\}$ is not continuous in the uniform operator topology by [1, Theorem (2.4)(ii)]. The group $\left\{\phi_{t}\right\}$ can be recovered from $\left\{T_{t}\right\}$, since for each $t \in \mathbf{R}, \phi_{t}=\left(T_{t} e_{1}\right) /\left(T_{t} e_{0}\right)$ on $D$. It is now evident that $M_{p}$ is one-to-one. On the other hand, if $p \neq 2$ and $\left\{U_{t}\right\} \in \Gamma_{p}$, then (see [1, Theorem (2.4)(i) and discussion leading up to it]) it follows from (0.1) that there are $\left\{\phi_{t}\right\} \in G$ and a function $\lambda(\cdot)$ from $\mathbf{R}$ into $C$ such that

$$
\left(U_{t} f\right)(z)=\lambda(t)\left[\phi_{t}^{\prime}(z)\right]^{1 / p} f\left(\phi_{t}(z)\right) \text { for } t \in \mathbf{R}, f \in H^{p}, z \in D .
$$

Taking $f$ equal to $e_{0}$ in (2.3) shows that $\lambda(\cdot)$ is continuous on $\mathbf{R}$. For each $s \in \mathbf{R}, U_{s}=\lambda(s) T_{s}$, where $\left\{T_{t}\right\} \in \Gamma_{p}$ corresponds to $\left(0,\left\{\phi_{t}\right\}\right)$ in (2.2). It follows that $\lambda(\cdot)$ is a continuous character of $\mathbf{R}$. This completes the proof.

REMARK. It is not difficult to show by example that the range of $M_{2}$ is a proper subset of $\Gamma_{2}$.

DEFinition. If $\left\{T_{t}\right\}=M_{p}\left(\omega,\left\{\phi_{t}\right\}\right)$, we shall call $\left\{\phi_{t}\right\}$ the conformal group associated with $\left\{T_{t}\right\}$, and $\omega$ the logarithmic index of $\left\{T_{t}\right\}$. We shall say that $\left\{T_{t}\right\}$ is of type (i), (ii), or (iii) according as $\left\{\phi_{t}\right\}$ is. Given any group $\left\{\phi_{t}\right\}$ of type (i) or (ii) with unique planar extension $\left\{\Phi_{w}\right\}$, we define $\left[\Phi_{w}^{\prime}(z)\right]^{1 / p}$ on $H_{\Phi} \times \bar{D}$ to be $\exp \left[(1 / p) L_{\Phi}(w, z)\right]$.

(2.4) THEOREM. If $1 \leqslant p<\infty$, and $\left\{T_{t}\right\}$ is a one-parameter group of $H^{p}$-isometries of type (i) or (ii), with associated conformal group $\left\{\phi_{t}\right\}$, then $\left\{T_{t}\right\}$ can be uniquely extended to a semigroup of bounded operators on $H^{p}$, $\left\{T_{w}\right\}, w \in H_{\Phi}$, such that $\left\{T_{w}\right\}$ is continuous on $H_{\Phi}$ in the strong operator topology and holomorphic on the interior of $H_{\Phi}$. If $\omega$ is the logarithmic index of $\left\{T_{t}\right\}$, then $\left\{T_{w}\right\}$ is given by:

$$
\left(T_{w} f\right)(z)=e^{i \omega w}\left[\Phi_{w}^{\prime}(z)\right]^{1 / p} f\left(\Phi_{w}(z)\right) \quad \text { for } f \in H^{p}, z \in D, w \in H_{\Phi} .
$$

Moreover, $\left\|T_{w}\right\| \leqslant e^{-\omega \operatorname{Im} w}$ for all $w \in H_{\Phi}$.

Proof. Uniqueness follows from the observation that, by the Schwarz reflection principle, a continuous complex-valued function on $H_{\Phi}$ analytic on the interior of $H_{\Phi}$ and vanishing on the real axis must vanish identically. For each $w \in H_{\Phi}$ it follows by a result of Gabriel $[5$, p. 117] that the right-hand side of (2.5) with the factor $e^{i \omega w}$ deleted defines a linear transformation $S_{w}$ of $H^{p}$ into $H^{p}$ such that $\left\|S_{w}\right\| \leqslant 1$. With the aid of (1.21) it is now clear 
that (2.5) defines a semigroup of bounded operators $\left\{T_{w}\right\}, w \in H_{\Phi}$, which extends $\left\{T_{t}\right\}, t \in \mathbf{R}$. To complete the proof it suffices to show that for each $f \in H^{p}$, and each continuous linear functional $G$ on $H^{p}, G\left(S_{w} f\right)$ is, as a function of $w$, continuous on $H_{\Phi}$ and analytic on the interior of $H_{\Phi}$. If $p>1$, then the evaluation functionals at points of $D$ span a norm-dense linear manifold in the dual space of $H^{p}$, and, since $\left\{S_{w}\right\}$ is uniformly bounded, it is enough to observe that if $G$ is evaluation at a point of $D$, then $G\left(S_{w} f\right)$ has the desired properties. If $p=1$, the proof reduces to the case where $f$ is a polynomial, and is then easily completed by passage to the boundary.

Definition. If $\left\{\phi_{t}\right\} \in G$ and $1 \leqslant p<\infty$, we shall denote $M_{p}\left(0,\left\{\phi_{t}\right\}\right)$ by $\left\{T_{t}^{(p, \phi)}\right\}$, or, when there is no danger of confusion, by $\left\{T_{t}^{(\phi)}\right\}$. Further, we denote the invariance polynomial of $\left\{\phi_{t}\right\}$ by $q_{\phi}$, and we define a closed linear operator $Q^{(p, \phi)}$, with both domain and range in $H^{p}$, as follows: its domain $D\left(Q^{(p, \phi)}\right)$ is $\left\{f \in H^{p}: q_{\phi} f^{\prime} \in H^{p}\right\}$, and

$$
\left(Q^{(p, \phi)} f\right)(z)=q_{\phi}(z) f^{\prime}(z)+\frac{1}{p} q_{\phi}^{\prime}(z) f(z) \quad \text { for } f \in D\left(Q^{(p, \phi)}\right), \quad z \in D .
$$

In a context where $p$ is fixed, we shall write $Q^{(\phi)}$ instead of $Q^{(p, \phi)}$.

(2.7) THEOREM. If $\left\{\phi_{t}\right\} \in G$ and $1 \leqslant p<\infty$, then the infinitesimal generator of $\left\{T_{t}^{(p, \phi)}\right\}$ is $Q^{(p, \phi)}$.

Proof. Let $A^{(\phi)}$ be the generator of $\left\{T_{t}^{(\phi)}\right\}$. If $f \in D\left(A^{(\phi)}\right)$ and $z \in$ $D$, then

$$
\left(A^{(\phi)} f\right)(z)=\left.\frac{d}{d t}\right|_{t=0}\left[\left(T_{t}^{(\phi)} f\right)(z)\right]
$$

and it follows that $Q^{(\phi)}$ extends $A^{(\phi)}$. If $\left\{\phi_{t}\right\}$ is of type (i), then $Q^{(\phi)}=$ $A^{(\phi)}$ by [1, Theorem (3.1)(i)]. If $\left\{\phi_{t}\right\}$ has the form (1.8) (resp., (1.9)), and if $\eta$ is any complex number, then it is readily verified that the function $F$ on $D$ whose value at each $z$ is given by

$$
(z-\alpha)^{2\left(p^{-1}-1\right)} \exp \{\alpha \eta / i c(z-\alpha)\}
$$

$$
\text { (resp., by } \left.(z-\alpha)^{1 / p-\eta / c-1}(z-\beta)^{1 / p+\eta / c-1}\right)
$$

has the property that for each analytic function $f$ on $D$

$$
\frac{d\left(F q_{\phi} f\right)}{d z}=-\left[n f-\left(q_{\phi} f^{\prime}+q_{\phi}^{\prime} f p^{-1}\right)\right] F \text { on } D \text {. }
$$

In particular, if $\eta=c$, and $f$ is in the null space of $c I-Q^{(\phi)}$, then there is a constant $K$ such that $f=K /\left(F q_{\phi}\right)$ on $D$. Since $1 /\left(F q_{\phi}\right)$ is not in $H^{p}$, we get that $c I-Q^{(\phi)}$ is one-to-one. Since the spectrum of the generator of a strongly continuous one-parameter group of Banach space isometries must be a subset of 
$i \mathbf{R}[2, \mathrm{VIII} .1 .11], c I-A^{(\phi)}$ maps $D\left(A^{(\phi)}\right)$ onto $H^{p}$. Because the one-to-one operator $c I-Q^{(\phi)}$ extends the surjective operator $c I-A^{(\phi)}$, we conclude easily that $D\left(Q^{(\phi)}\right)=D\left(A^{(\phi)}\right)$.

3. Spectral properties of $Q^{(p, \phi)}$. If $\left\{T_{t}\right\}=M_{p}\left(\omega,\left\{\phi_{t}\right\}\right)$, then the infinitesimal generator of $\left\{T_{t}\right\}$ is $i \omega+Q^{(p, \phi)}$. Thus, from the standpoint of spectral analysis, the study of such a generator reduces to the study of the corresponding operator $Q^{(p, \phi)}$. We shall denote the spectrum of an operator $A$ by $\Lambda(A)$.

(3.1) THEOREM. Let $\left\{\phi_{t}\right\} \in G$. For $1 \leqslant p<\infty$ we have:

(i) If $\left\{\phi_{t}\right\}$ is of type (i), with the representation (1.7), then: $Q^{(p, \phi)}$ has compact resolvent function; $\Lambda\left(Q^{(p, \phi)}\right)=\left\{i c\left(n+p^{-1}\right): n=0,1,2, \cdots\right\}$; for $n=0,1,2, \cdots, i c\left(n+p^{-1}\right)$ is an eigenvalue of $Q^{(p, \phi)}$, and the eigenmanifold corresponding to ic $\left(n+p^{-1}\right)$ is one-dimensional.

(ii) If $\left\{\phi_{t}\right\}$ is of type (ii), with the representation (1.8), then $\Lambda\left(Q^{(p, \theta)}\right)=$ $\{-i \lambda: \lambda c \geqslant 0\}$, and $2^{(p, \phi)}$ has no eigenvalues.

(iii) If $\left\{\phi_{t}\right\}$ is of type (iii), then $\Lambda\left(Q^{(p, \phi)}\right)=i \mathbf{R}$, and $Q^{(p, \phi)}$ has no eigenvalues.

Proof. In all cases we have $\Lambda\left(2^{(\phi)}\right) \subseteq i \mathbf{R}$ by virtue of Theorem (2.7). Assertion (i) is contained in [1, Theorem (3.1)] , and is listed above for comparison with (ii) and (iii). If $\left\{\phi_{t}\right\}$ is of type (ii) or (iii), and $\lambda \in \mathbf{R}$, then, just as in the proof of Theorem (2.7), an argument based on (2.8) (with $\eta=i \lambda$ in this instance) shows that $i \lambda-Q^{(\phi)}$ is one-to-one.

Next, let $\left\{\phi_{t}\right\}$ be of type (iii), with the representation (1.9). Let $\lambda \in \mathbf{R}$, and for each $w \in \mathrm{C}$ with $\operatorname{Re}(w)>-1 / p$, let $f_{w}$ be defined on $D$ by $f_{w}(z)=(z-\alpha)^{w}$. Then $f_{w}^{\prime}(z)=w(z-\alpha)^{-1} f_{w}(z)$, and we have that $f_{w} \in \mathcal{D}\left(Q^{(\phi)}\right)$. $\left[\left(i \lambda-Q^{(\phi)}\right) f_{w}\right](z)$

$$
\begin{aligned}
& =f_{w}(z)\left[i \lambda-\frac{w c}{\alpha-\beta}(z-\beta)-\frac{c}{p(\alpha-\beta)}(z-\alpha)-\frac{c}{p(\alpha-\beta)}(z-\beta)\right] \\
& =\frac{c}{\beta-\alpha}\left(w+\frac{2}{p}\right)(z-\alpha) f_{w}(z)-c\left(w-w_{0}\right) f_{w}(z), \text { where } w_{0}=-\frac{1}{p}+\frac{i \lambda}{c} .
\end{aligned}
$$

As $w \rightarrow w_{0}$ (with $\left.\operatorname{Re}(w)>-1 / p\right)$, $\left\|(z-\alpha) f_{w}\right\|_{\infty}=O(1)$, while $\left\|f_{w}\right\|_{p} \rightarrow$ $+\infty$. Thus $\left(\left\|\left(i \lambda-Q^{(\phi)}\right) f_{w}\right\|_{p} /\left\|f_{w}\right\|_{p}\right) \rightarrow 0$, and the proof of (iii) is complete.

If $\left\{\phi_{t}\right\}$ has the form (1.8), let $\left\{T_{w}\right\}, w \in H_{\Phi}$, be the extension of $\left\{T_{t}^{(\phi)}\right\}, t \in \mathbf{R}$, furnished by Theorem (2.4). In this instance $H_{\Phi}$ is $\{w \in \mathbf{C}$ : $c \operatorname{Im} w \leqslant 0\}$, and (since $\omega=0$ ) each $T_{w}$ is a contraction operator. Let $R$ be $\{w \in \mathrm{C}: \operatorname{Re}(w) \geqslant 0\}$. If $c>0$ (resp., $c<0$ ) define $\left\{U_{w}\right\}, w \in R$, by setting $U_{w}=T_{-i w}$ (resp., $U_{w}=T_{i w}$ ). By [6, Theorem 17.9.2] the infinites- 
imal generator $A$ of $\left\{U_{t}\right\}, t \geqslant 0$, is $(-i) Q^{(\phi)}$ (resp., $i Q^{(\phi)}$ ). Since, by [2, VIII.1.11], $\Lambda(A) \subseteq\{\mu \in \mathbf{C}: \operatorname{Re}(\mu) \leqslant 0\}$, we get that $\Lambda\left(Q^{(\phi)}\right) \subseteq\{-i \lambda: c \lambda \geqslant 0\}$. Conversely, if $c \lambda \geqslant 0$, for $t>-1 / p$ define $f_{t} \in H^{p}$ by setting $f_{t}(z)=$ $(z-\alpha)^{t} \exp (\lambda \alpha / c(z-\alpha))$. Then $f_{t} \in D\left(Q^{(\phi)}\right)$, and

$$
\left[\left(i \lambda+Q^{(\phi)}\right) f_{t}\right](z)=i c \bar{\alpha}\left(t+\frac{2}{p}\right)(z-\alpha) f_{t}(z) .
$$

As $t \rightarrow-1 / p$ from the right, clearly $\left\|(z-\alpha) f_{t}\right\|_{\infty}=O(1)$ and $\left\|f_{t}\right\|_{p} \rightarrow+\infty$. This completes the proof of the theorem.

REMARK. If $\left\{\phi_{t}\right\}$ is of type (ii) or (iii), then $Q^{(\phi)}$ does not have compact resolvent function, since $\Lambda\left(Q^{(\phi)}\right)$ is uncountable.

DEFINITION $_{b}$ For $1 \leqslant p<\infty$ and $\left\{\phi_{t}\right\} \in G$, let $\Delta^{(p, \phi)}$ be $\left\{\mu \in \Lambda\left(Q^{(p, \phi)}\right)\right.$ : the range of $\mu-Q^{(p, \phi)}$ is dense in $H^{p}$ \}.

(3.2) THEOREM. (i) If $\left\{\phi_{t}\right\}$ is of type (i), then $\Delta^{(p, \phi)}$ is empty for $1 \leqslant p<\infty$.

(ii) If $\left\{\phi_{t}\right\}$ is of type (ii), then $\Delta^{(p, \phi)}=\Lambda\left(Q^{(p, \phi)}\right)$ for $1<p<\infty$, and $\Delta^{(1, \phi)}=\{0\}$.

(iii) If $\left\{\phi_{t}\right\}$ is of type (iii), then $\Delta^{(p, \phi)}=\Lambda\left(Q^{(p, \phi)}\right)$ for $1<p<\infty$, and $\Delta^{(1, \phi)}$ is empty.

Proof. For $1 \leqslant p<\infty,\left\{\phi_{t}\right\} \in G$, and $\lambda \in \mathbf{R}$, it is clear that $i \lambda-$ $2^{(p, \phi)}$ generates a strongly continuous one-parameter group of isometries of $H^{p}$. The desired conclusions for $p>1$ are now immediate from Theorem (3.1) and the following general fact (see [2, VIII.7.2]): If $A$ generates a uniformly bounded strongly continuous semigroup of operators on a reflexive Banach space $X$, then $X^{*}$ is the direct sum of the null space of $A$ and the closure of the range of $A$.

To complete the proof of (i), let $\left\{\phi_{t}\right\}$ have the form (1.7), let $n$ be a nonnegative integer, and suppose $\left[i c(n+1)-Q^{(1, \phi)}\right] f=g$. It is easy to verify that on the region obtained by deleting $\tau$ from $D$

$$
\begin{aligned}
-\frac{\left(z-\bar{\tau}^{-1}\right)^{n+2} g(z)}{(z-\tau)^{n} q_{\phi}(z)} & =\frac{d}{d z}\left[\frac{\left(z-\bar{\tau}^{-1}\right)^{n+2}}{(z-\tau)^{n}} f(z)\right], & & \text { if } \tau \neq 0 \\
-\frac{g(z)}{z^{n} q_{\phi}(z)} & =\frac{d}{d z}\left(\frac{f(z)}{z^{n}}\right), & & \text { if } \tau=0 .
\end{aligned}
$$

The right-hand side of each equation in (3.3) has a vanishing contour integral around any circle $|z|=r,|\tau|<r<1$. Thus we get for $\tau \neq 0$ (resp., $\tau=0$ ) $\left(\left(z-\bar{\tau}^{-1}\right)^{n+1} g(z)\right)^{(n)}(\tau)=0$ (resp., $\left.g^{(n)}(0)=0\right)$. Since the range of $i c(n+1)-$ $Q^{(1, \phi)}$ is contained in the null space of a nonzero continuous linear functional on $H^{1}$, the desired conclusion follows.

Next let $\left\{\phi_{t}\right\}$ have the form (1.8). If $\lambda c>0$ and $g=-\left(i \lambda+Q^{(1, \phi)}\right) f$, 
then by (2.8) there is a constant $K$ such that for all $z \in D$

$$
f(z)=-\frac{1}{F(z) q_{\phi}(z)}\left[\int_{0}^{z} g(\xi) F(\xi) d \xi+K\right],
$$

where $F(z)=\exp (-\lambda \alpha / c(z-\alpha)$ ) (we shall regard $F$ as being defined on $\bar{D} \backslash\{\alpha\}$ by this formula). We observe that $|F(z)|=\exp (\lambda / 2 c)$ for $z \in C, z \neq$ $\alpha$. However, $F$ does not belong to $H^{\infty}(D)$, whereas the reciprocal of $F$ does. As $z$ approaches radially any point $z_{0}$ of $C \backslash\{\alpha\}$, the integral in (3.4) tends to a limit (denoted $\left.\int_{0}^{z} g(\xi) F(\xi) d \xi\right)$. Since $g\left(e^{i \theta}\right) F\left(e^{i \theta}\right)$ is in $L^{1}$ of $C$, it is easy to see that $\int_{0}^{z} g(\xi) F(\xi) d \xi$ has one-sided limits as $z \rightarrow \alpha$ on $C$. Because $f\left(e^{i \theta}\right)$ is in $L^{1}$ of $C$, both one-sided limits must be $-K$. It follows that $g$ is in the null space of the continuous linear functional $\dot{y}(\cdot)$ on $H^{1}$-defined by $y(h)=$ $\int_{|\xi|=1} h(\xi) F(\xi) d \xi$. Note that since $1 / F$ does not have an inverse in the algebra $H^{\infty}(D)$, the restriction of $F$ to $C$, which is the reciprocal of the boundary function of $1 / F$, cannot be in $H^{\infty}(C)$. However, if $y$ were identically zero, this restriction of $F$ would be in $H^{\infty}(C)$. It follows that $-i \lambda \notin \Delta^{(1, \phi)}$ for $c \lambda>$ 0 . On the other hand, if $h$ is analytic on $D$ with $h(z)=O\left(|z-\alpha|^{2}\right)$, then, as a consequence of Hardy's inequality, $h$ has a primitive $G$ on $D$ such that $G(z) \rightarrow 0$ as $z \rightarrow \alpha$. It is easy to see that $G(z) / q_{\phi}(z)=O(|z-\alpha|)$ and $Q^{(1, \phi)}\left(G / q_{\phi}\right)=h$. Since $(z-\alpha)$ is outer, $O \in \Delta^{(1, \phi)}$.

Suppose next that $\left\{\phi_{t}\right\}$ has the form (1.9), that $\lambda \in \mathbf{R}$, and that $\left(i c \lambda-Q^{(1, \phi)}\right) f=g$. As before, equation(3.4) holds on $D$, and $F$ now has the form $(z-\alpha)^{-i \lambda}(z-\beta)^{i \lambda}$. In this instance both $F$ and its reciprocal are in $H^{\infty}$, and, by virtue of Hardy's inequality, the integral appearing in (3.4) has, as a function of $z$, a continuous extension to $\bar{D}$. It is easy to see that we must have

$$
\int_{0}^{\alpha} g(\xi) F(\xi) d \xi=\int_{0}^{\beta} g(\xi) F(\xi) d \xi=-K .
$$

Thus $\int_{\alpha}^{\beta} g(\xi) F(\xi) d \xi=0$ (either arc of $C$ from $\alpha$ to $\beta$ may be used for this path integral by [3, Theorem (3.6)]). (It is easy to see that the range of (ic $\lambda$ $\left.2^{(1, \phi)}\right)$ contains $\left\{G \in \bigcup_{1<p \leqslant \infty} H^{p}: \int_{\alpha}^{\beta} G(\xi) F(\xi) d \xi=0\right\}$; however, we do not need this fact, and omit its proof.) Let us define a continuous linear functional $y(\cdot)$ on $H^{1}$ by the formula $y(h)=\int_{\alpha}^{\beta} h(\xi) F(\xi) d \xi$. Note that $y(1 / F)=\beta-\alpha$ $\neq 0$. This completes the proof of the theorem.

4. Concluding remarks concerning ranges. In this section we shall examine the range of $\left(\mu-Q^{(p, \phi)}\right)$, where $\mu \in \Delta^{(p, \phi)}$. Our aim will be to find a (moreor-less concretely described) dense subset of $H^{p}$ which is also a subset of the range of $\left(\mu-2^{(p, \phi)}\right)$. For $p=1$ this setting occurs if and only if $\left\{\phi_{t}\right\}$ is of type (ii) and $\mu=0$; for this case a specific dense subset of $H^{1}$ which is also a subset 
of the range of $2^{(1, \phi)}$ was found in the proof of Theorem (3.2).

(4.1) THEOREM. Suppose that $1<p<\infty$, and $\left\{\phi_{t}\right\}$ is of type (iii) with the representation (1.9). For each $\lambda \in \mathbf{R}$,

$$
\left\{g \in \bigcup_{p<s \leqslant \infty} H^{s}: \int_{\alpha}^{\beta} g(\xi)(\xi-\alpha)^{-i \lambda}(\xi-\beta)^{i \lambda}[(\xi-\alpha)(\xi-\beta)]^{(1 / p)-1} d \xi=0\right\}
$$

is dense in $H^{p}$, and is a subset of the range of $\left(i c \lambda-Q^{(p, \phi)}\right)$.

Proof. We note that in the above contour integral, we are multiplying $g$ by the function $F$ appearing in (2.8) (for $\eta=i c \lambda$ ). Let $g \in H^{p_{1}}$ for some $p_{1}>p$, and observe that $F g \in H^{1+\delta}$ for some $\delta>0$. If $\int_{\alpha}^{\beta} g(\xi) F(\xi) d \xi=0$, then the function of $z \in D \int_{\alpha}^{z} g(\xi) F(\xi) d \xi\left(=\int_{\beta}^{z} g(\xi) F(\xi) d \xi\right)$ is $O\left(|z-\alpha|^{\delta /(1+\delta)}\right)$ and $O\left(|z-\beta|^{\delta /(1+\delta)}\right)$. From this we see that $-\left(F(z) q_{\phi}(z)\right)^{-1} \int_{\alpha}^{z} g(\xi) F(\xi) d \xi$ is in $H^{p}$. By (2.8) $\left(i c \lambda-Q^{(p, \phi)}\right)$ sends the latter function into $g$.

To complete the proof let $X$ be the normed linear space $\bigcup_{p<s<\infty} H^{s}$ (with $H^{p}$-norm). Define the linear functional $G$ on $X$ by $G(g)=\int_{\alpha}^{\beta} g(\xi) F(\xi) d \xi$. We show that the kernel of $G$ is dense in $X$ by proving that $G$ is not continuous. For $t>-1 / p$, define $f_{t} \in X$ by

$$
f_{t}(z)=(z-\alpha)^{i \lambda}(z-\beta)^{-i \lambda}(z-\alpha)^{t}(z-\beta)^{1-1 / p} .
$$

Then

$$
G\left(f_{t}\right)=\int_{\alpha}^{\beta}(\xi-\alpha)^{t+p^{-1}-1} d \xi=(\beta-\alpha)^{t+p^{-1}}\left(t+p^{-1}\right)^{-1} .
$$

But as $t \rightarrow-1 / p,\left\|f_{t}\right\|_{p}=O\left(\left(t+p^{-1}\right)^{-1 / p}\right)$.

The following lemma will be useful in studying the type (ii) case.

(4.2) Lemma. If $f \in H^{\infty}, \alpha \in C$, and $\lambda \leqslant 0$, then

$$
\left|\int_{-\alpha}^{z} e^{\lambda \alpha /(\xi-\alpha)} f(\xi) d \xi\right|=O\left(\left|e^{\lambda \alpha /(z-\alpha)}\right|\right), \text { for }|z|<1 .
$$

Proof. For convenience we shall, without loss of generality, take $\alpha$ equal to 1. Integrate from -1 along $[-1,1]$ to the point $\xi_{0}$ on the circle $\operatorname{Re} 1 /(\xi-1)=\operatorname{Re} 1 /(z-1)$, and then along the minor arc of this circle from $\xi_{0}$ to $z$. Since $\lambda /(x-1)$ increases on $[-1,1)$, the absolute value of the first integral does not exceed $2\|f\|_{\infty}\left|e^{\lambda /(z-1)}\right|$. The absolute value of the second integral does not exceed $\pi\|f\|_{\infty}\left|e^{\lambda /(z-1)}\right|$.

(4.3) THEOREM. Suppose that $1<p<\infty$, and $\left\{\phi_{t}\right\}$ is of type (ii) with the representation (1.8). For each $\lambda \leqslant 0$, 


$$
\begin{aligned}
& \left\{g \in H^{\infty}: g(z)=O\left(|z-\alpha|^{2}\right)\right. \text { and } \\
& \left.\qquad \int_{|\xi|=1} g(\xi)(\xi-\alpha)^{2\left(p^{-1}-1\right)} e^{(\lambda \alpha) /(\xi-\alpha)} d \xi=0\right\}
\end{aligned}
$$

is dense in $H^{p}$, and is a subset of the range of $\left(i c \lambda-Q^{(p, \phi)}\right)$.

Proof. Let $N$ be the set in the statement of the theorem, and observe that in the (absolutely convergent) contour integral used to define $N, g$ is multiplied by the function $F$ appearing in (2.8) (for $\eta=i c \lambda$ ). For any $g \in \mathbb{N}$, $g(z)(z-\alpha)^{2((1 / p)-1)}$ is in $H^{\infty}(D)$. Define $G$ on $D$ by

$$
G(z)=\int_{-\alpha}^{z} g(\xi) F(\xi) d \xi+\int_{\alpha}^{-\alpha} g(\xi) F(\xi) d \xi
$$

(by assumption on $g$, the latter integral is the same along either semicircle from $\alpha$ to $-\alpha$ ). By Lemma (4.2),

$$
\frac{G(z)}{F(z) q_{\phi}(z)}=O\left(|z-\alpha|^{-2 / p}\right) \text { for }|z|<1 .
$$

Clearly, then, $G / F q_{\phi}$ is in $H^{1 / 3}$. The boundary function of $G / F q_{\phi}$ is

$$
\frac{1}{F q_{\phi}}\left(\int_{\alpha}^{z} g(\xi) F(\xi) d \xi\right)
$$

where the integral is taken along either arc of $C$ from $\alpha$ to $z$. It is easy to see that this boundary function is $O(|z-\alpha|)$ for $|z|=1$, and it follows by [3, Theorem (2.7)] that $G / F q_{\phi}$ is in $H^{\infty}(D)$. By $(2.8),\left(i c \lambda-Q^{(p, \phi)}\right)\left(-G / F q_{\phi}\right)=g$.

Let $X$ be the set of all analytic functions on $D$ which are $O\left(|z-\alpha|^{2}\right)$. If $\lambda=0$, then by the Cauchy integral theorem $N=X$, and the density of $N$ is obvious in this case. If $\lambda<0$, we regard $X$ as a normed linear space with the $H^{p}$-norm, and we complete the proof of the theorem by showing that the linear functional $f \mapsto \int_{|\xi|=1} f(\xi) F(\xi) d \xi$ is not continuous on $X$. Without loss of generality we take $\alpha=1$. If the functional were continuous, there would be a function $W$ in $L^{p^{\prime}}(d \theta)$ (where $p^{\prime}$ is conjugate to $p$ ) such that

$$
\int_{|\xi|=1} g(\xi)(\xi-1)^{2} F(\xi) d \xi=\int_{|\xi|=1} g(\xi)(\xi-1)^{2} W(\xi) d \xi \quad \text { for all } g \in H^{\infty} .
$$

It follows, since $(\xi-1)^{2} F(\xi)$ and $(\xi-1)^{2} W(\xi)$ are integrable, that there is a function $h \in H^{1}$ such that $(\xi-1)^{2} F(\xi) d \xi=(\xi-1)^{2} W(\xi) d \xi+h(\xi) d \xi$. This gives

$$
(\xi-1)^{2 / p} d \xi=e^{-\lambda /(\xi-1)}(\xi-1)^{2} W(\xi) d \xi+e^{-\lambda /(\xi-1)} h(\xi) d \xi .
$$


Multiply (4.4) by $(2 \pi i)^{-1}(\xi-r)^{-3}, 0<r<1$, and then integrate both sides around $C$. The left side is $p^{-1}((2 / p)-1)(r-1)^{2((1 / p)-1)}$. For any $b>0$, the second term on the right is $O\left((1-r)^{b}\right)$, while standard estimates show that the first term on the right is $O\left((1-r)^{-1+1 / p}\right)$. By letting $r \rightarrow 1$ we obtain the desired contradiction if $p \neq 2$. If $p=2$, multiply (4.4) by $(2 \pi i)^{-1}(\xi-r)^{-3}(\xi-1)^{1 / 4}$. As before, we obtain a contradiction.

\section{REFERENCES}

1. E. Berkson and H. Porta, Hermitian operators and one-parameter groups of isometries in Hardy spaces, Trans. Amer. Math. Soc. 185 (1973), 331-344.

2. N. Dunford and J. T. Schwartz, Linear operators. I: General theory, Pure and Appl. Math., vol. 7, Interscience, New York, 1958. MR 22 \#8302.

3. P. Duren, Theory of $H^{p}$ spaces, Pure and Appl. Math., vol. 38, Academic Press, New York, 1970. MR 42 \#3552.

4. F. Forelli, The isometries of $H^{p}$, Canad. J. Math. 16 (1964), 721-728. MR 29 \#6336.

5. R. Gabriel, Some results concerning the integrals of moduli of regular functions along certain curves, J. London Math. Soc. 2 (1927), 112-117.

6. E. Hille and R. S. Phillips, Functional analysis and semi-groups, rev. ed., Amer. Math. Soc. Colloq. Publ., vol. 31, Amer. Math. Soc., Providence, R. I., 1957. MR 19, 664.

7. N. Levinson and R. M. Redheffer, Complex variables, Holden-Day, San Francisco, Calif., 1970. MR 42 \#6193.

DEPARTMENT OF MATHEMATICS, UNIVERSITY OF ILLINOIS, URBANA, ILLINOIS 61801 